\title{
Reducing hERG Toxicity Using Reliable hERG Classification Model and Fragment Grow Model
}

Yan Yang ${ }^{\mathrm{a}, 1}$, Yanmin Zhang, ${ }^{\mathrm{a}, 1}$, Yihang Zhang ${ }^{\mathrm{a}}$, Xingye Chen ${ }^{\mathrm{a}}$, Yi Hua ${ }^{\mathrm{a}}$, Guomeng

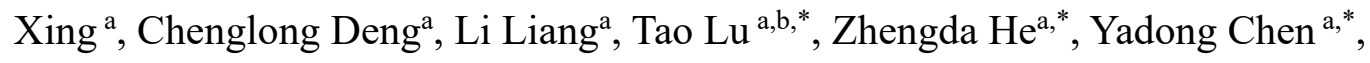
Haichun Liu ${ }^{\text {a,* }}$

${ }^{a}$ Laboratory of Molecular Design and Drug Discovery, School of Science, China Pharmaceutical University, 639 Longmian Avenue, Nanjing 211198, China

${ }^{\mathrm{b}}$ State Key Laboratory of Natural Medicines, China Pharmaceutical University, 24 Tongjiaxiang, Nanjing 210009, China

${ }^{1}$ The authors contributed equally to this work and should be considered as co-first authors.

\begin{abstract}
Drug-induced cardiotoxicity has become one of the major reasons leading to drug withdrawal in past decades, which is closely related to the blockade of human Ether-ago-go-related gene (hERG) potassium channel. Developing reliable hERG predicting model and optimizing model can greatly reduce the risk faced in drug discovery. In this study, we constructed eight hERG classification models, the best of which shows desirable generalization ability on low-similarity clinical compounds, as well as advantages in perceiving activity gap caused by small structural changes. Furthermore, we developed a hERG optimizer based on fragment grow strategy. Results reveal that after reinforcement learning, our model can provide reasonable optimizing direction to reduce hERG toxicity, especially when hERG risk is corresponding to lipophilicity, basicity and pi-pi interactions. We also prove its usage in helping chemists quickly pick out core fragments and fix on the region to be optimized. Overall, we demonstrate our
\end{abstract}


model as a promising tool for medicinal chemists in hERG optimization attempts. 


\section{Introduction}

Cardiotoxicity has become one of the major causes leading to drug failure, accounting for $18.8 \%$ of withdrawal between 1990 and $2010^{1}$. The blockade of a cardiac potassium channel encoded by human Ether-a-go-go-related gene (hERG) is responsible for the drug-induced cardio disorder, which is associated with a prolongation of QT-interval in the electrocardiogram (ECG) ${ }^{2-3}$. Assessment of potential hERG-related cardiotoxicity has become a critical step in the drug discovery pipeline ${ }^{4}$. Many established experimental methods have been developed to measure hERG binding affinity in the past such as patch clamp assays, fluorescence-based assays and radioligand binding assays $^{5}$, providing reliable statistics to medicinal chemists. However, it is timeconsuming and expensive to test every candidate in the early stage. Therefore, it is of great use to build in silico model for the prediction of hERG risk.

Apart from hERG predicting, another important question is how to optimize compounds when they are found to be hERG channel blockers. It will greatly save the labor if the computer can suggest some structures with low hERG risk but high similarity to the original structures. Actually, this task is what we called propertyoriented molecule generation. Previous works have proposed various approaches to optimize a certain property of a compound. Marcus Olivecrona and co-workers introduced a SMILES-based model to generate active compounds against the dopamine receptor type 2 (DRD2) using Policy Gradient, a reinforcement learning method ${ }^{6}$. Same technique was implemented into the network of Reinforcement Learning for Structural Evolution (ReLeaSE) by Mariya Popova and co-workers ${ }^{7}$. Wengong Jin and coworkers $^{8}$ employed a junction tree based network (JT-VAE) to automatic design molecules with specific chemical properties. Its performance was demonstrated to be better than other VAE-based methods. Jiaxuan You and co-workers ${ }^{9}$ adopted a Graph Convolutional Policy Network (GCPN) to perform goal-directed molecule generation, which uses graph information and Proximal Policy Optimization (PPO) algorithm. It can obtain $61 \%$ improvement compared with JT-VAE in the task of optimizing 
penalized $\log$ P. Łukasz Maziarka and co-workers proposed a CycleGAN-based model that can generate optimized compounds with desired property and high structural similarity to the original ones ${ }^{10}$. It was reported to significantly outperform previous results in terms of optimizing penalized $\log \mathrm{P}$ of drug-like molecules. Instead of using network based on SMILES or molecular graph, Zhenpeng Zhou and co-workers ${ }^{11}$ chose to directly modify the molecule by adding or removing atoms and bonds, which is called Molecule Deep Q-Networks (MolDQN), another reinforcement learning based method. MolDQN can generate better result than JT-VAE and comparable result to GCPN. Despite a wide range of options, few methods can specify the optimizing region. Sometimes chemists may desire keeping core fragments while growing new fragments on specific growing sites. However, approaches such as ReLeaSE, JT-VAE and CycleGAN are only suitable for de no vo drug design. Users cannot define which fragment to be kept in the output structures. As for GCPN and MolDQN, they can start from a given fragment. But as they automatically define growing sites, sometimes it might reduce attention on the optimization in specific area that chemists care about.

Herein, we introduce a fragment growing network that enable users to keep particular substructures and optimize a user-defined region. Reinforcement learning is applied to increase the network's ability to generate low-hERG-risk molecules. In order to make a reliable reward function, we first construct eight hERG classification models to predict hERG toxicity of compounds. The best model shows desirable performance on different datasets and different level of tasks, especially strong generalization capability on clinical external set and best competence in recognizing the activity difference in optimization cases. Then the fragment growing network is built, which can keep userdefined substructures in the generated structures. The basic framework is a traditional sequence-based generator (Recurrent Neural Network, RNN). However, the kept parts, which are imprecisely but understandably called "core fragments" in our study, are encoded by Gated Graph Neural Networks (GGNN) ${ }^{12}$ to facilitate fragment grow. The combination model of GGNN and RNN shows superior result to naive RNN in terms of validity and synthetic accessibility for the generated structures. We also demonstrate 
the application of the model in suggesting potential core fragments to chemists. The grow model, assisted by reinforcement learning, is then tested on five cases extracted from the literature ${ }^{3}$. Each case represents a smart strategy to lower hERG binding affinity concluded by experienced chemists. The robust hERG classification model work as a part of reward function for reinforcement learning. By comparing the generated molecules with chemists' final optimized compounds, we demonstrate the potential of our model in providing chemists reasonable direction for hERG optimization.

\section{Materials and Methods}

\section{Constructing hERG Classifying Model}

Data Collection and Process. Data from Chembl (https://www.ebi.ac.uk/chembl/, Chembl Target ID: Chemb1240), PubChem (https://pubchem.ncbi.nlm.nih.gov/, NCBI Protein: Q12809) and literatures ${ }^{13-15}$ was merged. Compounds without $\mathrm{IC}_{50}$ or Ki label were removed from the merged collection. All units were standardized to $\mu \mathrm{M}$. Then the data was sorted into two classes according to their activity, with $\mathrm{IC}_{50} / \mathrm{Ki}$ equal or less than $10 \mu \mathrm{M}$ labeled as blockers and $\mathrm{IC}_{50} / \mathrm{Ki}$ equal or greater than $30 \mu \mathrm{M}$ labeled as nonblockers according to Hongmao Sun's work ${ }^{16}$. Compounds with conflicting activity labels were abandoned to ensure the optimal data consistency. After that, structures that had entered clinical phases were separated as external validation dataset for evaluation. The remaining data was randomly divided into training set and test set (4:1) to constructing predicting model. The information of each dataset can be seen in Table 1 .

Table 1. Information of Datasets used for hERG Classification Model

\begin{tabular}{cccccc}
\hline \multicolumn{2}{c}{ Training Set } & \multicolumn{2}{c}{ Test Set } & External Validation Set \\
\hline Blockers & Non-blockers & Blockers & Non-blockers & Blockers & Non-blockers \\
5893 & 3382 & 1466 & 854 & 142 & 109 \\
\hline
\end{tabular}

Descriptors Calculation and Selection. It has been reported that some physicochemical 
properties of hERG channel blockers including lipophilicity, acidity or basicity, the pipi interaction of aromatic rings and the number of rotatable bonds are related to high hERG risk $^{17}$. Strategies directing at improving these four properties have been successfully applied to mitigate hERG activity ${ }^{3}$, indicating their importance in hERG model building. Therefore, related properties should be calculated and selected to build hERG model. The process was conducted as follows: Compounds were firstly added hydrogen and optimized in MMFF94 force field using RDKIT package (http://www.rdkit.org/, version: 2018.09.1.0) in Python3.6. Then 115 2D properties and 1024 ECFP4 bits calculated by RDKIT, together with two properties calculated by Mordred $^{18}$ (nAcid, nBase) for the training set were incorporated. Among these features, MolLogP is corresponding to lipophilicity; NumAromaticRings (NAroR) is related to possibility of forming pi-pi interaction; the number of rotatable bonds is represented by NumRotatableBonds (NROT). nAcid and nBase might be related to the acidity or basicity, so we also put them into the property pond and further property selection. The incorporated 1,141 descriptors were reduced to 162 by low variance filter (threshold $=0.1$ ) and high correlation filter (threshold $=0.9$ ), and they were subsequently selected by Non-dominated Sorting Genetic Algorithm-II (NSGA-II) ${ }^{2}$, which distinguished 61 out of 162 features to reach the best five-fold cross-validated Kappa of training set. MolLogP, nAcid and nBases were selected by NSGA-II, demonstrating their relative importance in hERG model. NAroR and NROT are unselected. In order to increase the reliability of our model, we manually added them into the final feature set. The selected descriptors can be found in Table S1.

NSGA-II is a multi-objective genetic algorithm based on a non-dominated set, which have been applied to select features in the previous study ${ }^{2}$. It started with random initialization of 162-bit binary individuals, with each bit accounting for the presence (on-bit) or absence (off-bit) of a corresponding feature. In our study, the population size was set as 30. In each population, crossover was defined as random exchange of 30 bits between two individuals, after which one-point mutation occurred on their offspring when the random mutation probability was greater than 0.5 . Crossover and mutation 
generated another 30 individuals for each population, doubling the size to a total of 60 . Individuals were then ranked and selected according to their dominance levels, which were decided by their total count of features and five-fold cross-validated Kappa of the SVM model they generated (parameters of SVM were set as default). The procedure repeated over 100 times and finally found out the minimal number of features to reach the highest five-fold cross-validated Kappa.

Model Construction. Eight widely used methods were adopted to build hERG classification model, including Support Vector Machine (SVM), Random Forest (RF), Extremely Randomized Trees (ExtraTree), Adaptive Boosting (Adaboost), Gradient Boosted Decision Trees (GBDT), Exterme Gradient Boosting (XGBoost), Light Gradient Boosting Machine (LightGBM) and Deep Neural Networks (DNN). Scikitlearn (https://scikit-learn.org/, $\quad$ version: 0.23 .1$), \quad$ XGBoost (https://github.com/dmlc/xgboost, $\quad$ version: 1.1.1), LightGBM (https://github.com/microsoft/LightGBM,,$\quad$ version:2.3.1) and pyTorch (https://pytorch.org/, version:1.3.1) package were used to build models. Grid search was applied to explore the best combination of parameters for highest five-fold cross validated accuracy, while class weight for non-blockers was fixed as the ratio of blockers to non-blockers uniformly. The detailed description of other parameters can be seen in Supporting Information.

Model Evaluation. The obtained eight models were assessed using a variety of measures, which contained Area Under the ROC Curve (AUC), Accuracy (ACC), Balanced Accuracy (BAC), Matthews Correlation Coefficient (MCC), Cohen's Kappa (Kappa), F1_Score (F1), precision, recall (sensitivity), specificity (Supporting Information). The absolute values of all these metrics range from 0 to 1 , with 1 representing the best and 0 representing the worst performance. 10 -fold metrics of training set, together with metrics of test set and extra validation set were calculated and compared to select top models used for further validation.

To investigate the selected models' practicability on correctly predicting new 
structures, we scaled the extra validation set according to their Tanimoto similarity /ECFP4 to training set. Seven subsets were thus created, with similarity value $\geq 0.8$, $<0.8,<0.7,<0.6,<0.5,<0.4$ and $<0.3$ respectively. The subset with similarity greater than 0.8 contains structures that are highly similar to the training set. Therefore, it is no surprising it will generate a good result. By contrast, it will be more convincing that if subsets with low similarity can also produce a good performance. We believe that structures with similarity below 0.3 are new enough compared with the training set. BAC for each subset was displayed for the chosen models. This step generated best three models with best generalization ability.

Validation on Optimization Cases. Generally speaking, it is more challenging for a model to recognize "activity cliffs", which means classifying structures with huge gap of hERG binding affinity but subtle structural differences into the right groups. Given that activity cliffs usually happen in chemists' compound optimization cases, herein, we tested our three best models on seven cases provided by previous literature ${ }^{3}$. Each case is constituted by two compounds with different $\mathrm{IC}_{50}$ values, marked by $\mathbf{a}$ and $\mathbf{b}$ respectively. Compounds marked by a refer to the original structures that suffered from high hERG risk $(<10 \mu \mathrm{M})$, and compounds marked by $\mathbf{b}$ are optimized structures with much lower hERG binding affinity $(>30 \mu \mathrm{M})$ corresponding to $\mathbf{a}$. The original structures in case $\mathbf{1}$ will be named compound 1a, while the optimized will be named compound 1b and so on. The detailed information of these cases can be seen in Table 2. All the molecules in these case do not appear in the training set. We compared the predicting accuracy of our three pre-selected models on these cases and finally picked the best one. 
Table 2. Seven Optimization Cases Extracted from Literature

\begin{tabular}{|c|c|c|c|c|c|c|c|}
\hline $\begin{array}{l}\text { Case } \\
\text { Index }\end{array}$ & hERG Channel Blockers & ID & $\begin{array}{c}\text { hERG IC }_{50} \\
(\mu \mathrm{M})\end{array}$ & hERG Improved & ID & $\begin{array}{c}\text { hERG IC }_{50} \\
(\mu \mathrm{M})\end{array}$ & $\begin{array}{c}\text { Optimization } \\
\text { Strategy }\end{array}$ \\
\hline 1 & & $1 \mathrm{a}$ & 1.4 & & $1 \mathrm{~b}$ & $>33$ & $\begin{array}{l}\text { Decreasing } \\
\text { lipophilicity }\end{array}$ \\
\hline 2 & & $2 \mathrm{a}$ & 8.9 & & $2 b$ & $>30$ & $\begin{array}{l}\text { Decreasing } \\
\text { lipophilicity }\end{array}$ \\
\hline 3 & & $3 a$ & 8.1 & & $3 b$ & $>198$ & $\begin{array}{c}\text { Lowering } \\
\text { basicity }\end{array}$ \\
\hline 4 & & $4 a$ & 4.19 & & $4 \mathrm{~b}$ & 39 & $\begin{array}{c}\text { Magnifying } \\
\text { rigidity }\end{array}$ \\
\hline 5 & & $5 \mathrm{a}$ & 6.7 & & $5 b$ & $>80$ & $\begin{array}{l}\text { Altering pi-pi } \\
\text { interactions }\end{array}$ \\
\hline 6 & & $6 a$ & 6.3 & & $6 \mathrm{~b}$ & $>100$ & $\begin{array}{c}\text { No particular } \\
\text { regularity }\end{array}$ \\
\hline 7 & & $7 a$ & 4.6 & & $7 b$ & 68 & $\begin{array}{c}\text { No particular } \\
\text { regularity }\end{array}$ \\
\hline
\end{tabular}

\section{Fragment Grow Network}

Model Overview. The main intention of our model is to optimize compound while keep substructures that chemists want to preserve because of activity demand or other reasons. Thus, we adopted a 'fragment grow' strategy that requires our model to learn how to generate target fragments on a given 'core fragment' instead of generating brand new molecules. In the training stage, we firstly broke a compound into two fragments. 
One fragment is called 'core fragment', as we assume that it is what chemists think is important and will not be changed. The other is taken as 'source fragment', which borrows the word 'source' from 'source sequence' in Natural Language Processing (NLP). As shown in Figure 1, The model mainly learns grammatical rules from the standardized SMILES of 'source fragment' using an encoder-decoder network. On the other hand, it also absorbs the graph information of 'core fragment' using GGNN to give the decoder an extra context. In other words, the model will 'understand' the scene, which means it can recognize which 'core fragment' it is supposed to grow on, which growing site it is supposed to start with and the surrounding information of the growing site. The two networks allow the model to reasonably grow target fragments based on the core fragments. Finally, target fragments are linked with core fragments on the growing site, generating the whole structures. Considering the existence of both GGNN and RNN network, we brief it as GGNN-RNN model.

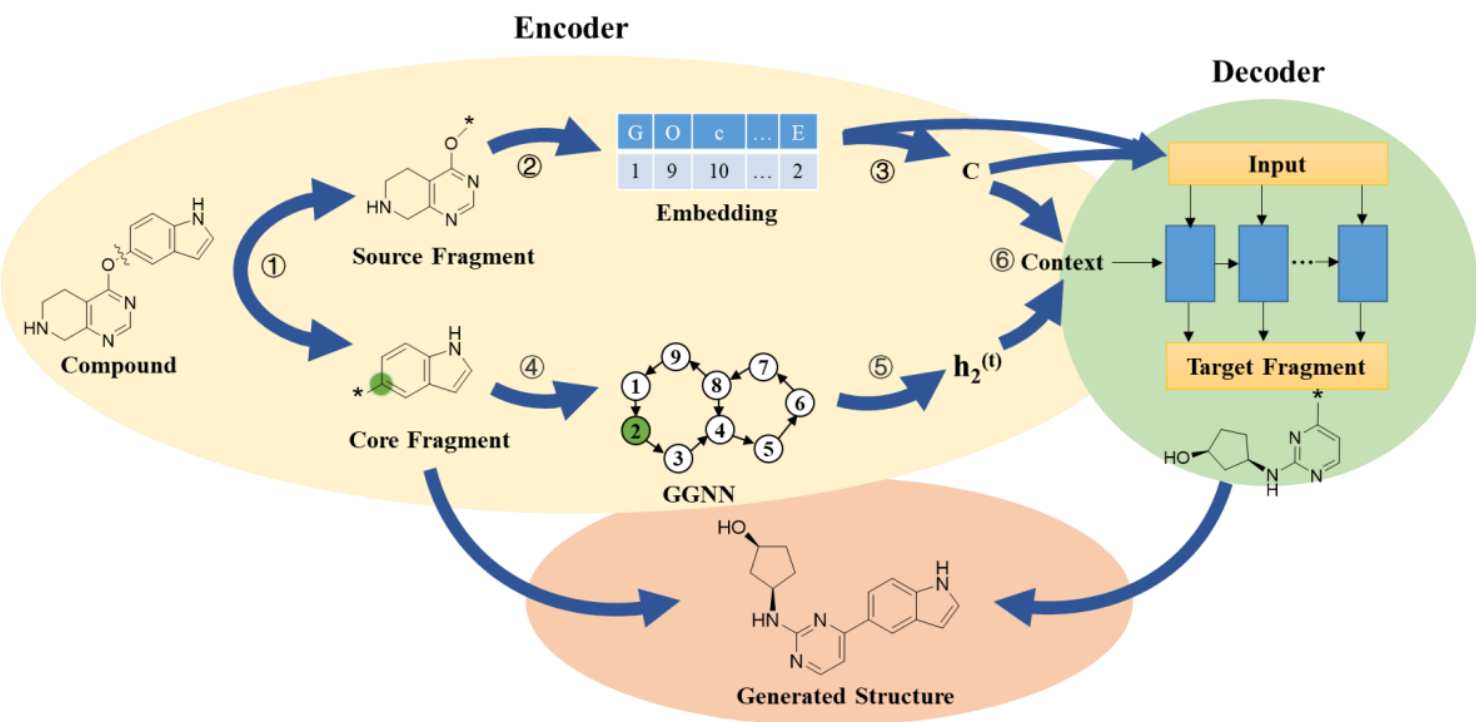

Figure 1. Brief Workflow of GGNN-RNN. The network adopts an encoder-decoder structure. The encoder involves six steps: (1) The compound is broken into two pieces. (2) The SMILES of source fragment is uniformed and processed using one hot method.

(3) The processed information of the source fragment is encoded as a variable C. (4) The core fragment is represented as graph, where each node receives and send out message along edges that connecting nodes. (5) The message of the growing site (the node 2) at step $t$ is extracted as $\mathbf{h}_{2}{ }^{(t)}$ (6) $\mathbf{C}$ and $\mathbf{h}_{2}{ }^{(\mathbf{t})}$ is combined as the total context of 
the decoder. The decoder produces target fragments using the information of the source target and the context. Finally, the core fragment is connected with the target fragment and generate the whole structure.

Data Preparation. Active kinase inhibitors with $\mathrm{IC}_{50} / \mathrm{Ki}$ less than $10 \mu \mathrm{M}$ from Chembl database were collected, which belong to a total of 612 kinases target (supporting information). Inorganic structures and duplicates were removed. Structures containing phosphorus were also removed, as it is relatively less common use in drugs. However, users can train their own model if needed. Molecule weight was controlled between 100 and 800, after which 103, 292 cleaned kinase inhibitors were obtained. We broke these inhibitors by cutting on one single bond a time (bonds outside the ring but connecting with the ring), which generated many fragment pairs for each molecule. The larger fragments were used as 'core fragment', and the smaller were taken as 'source fragment', whose molecule weight was limited to no more than $350 \mathrm{Da}$. Both fragments carried a '*' to indicate the breaking site/growing site. To reduce the computation cost, only three pairs for each molecule were randomly selected and gathered as our model input.

Source fragments were standardized using RDKIT, which always puts the growing site ' $*$ ' at the beginning of the SMILES. For example, SMILES ' $\mathrm{O}=\mathrm{C}([*])[\mathrm{C} @ \mathrm{H}] 1 \mathrm{NCCCC1}$ ' will be unified to '* $\mathrm{C}(=\mathrm{O})[\mathrm{C} @ @ \mathrm{H}] 1 \mathrm{CCCCN} 1$ ' in RDKIT. In this way, we can easily replace '*' with a ' $G$ ' to tell the generator a sequence of fragment is to begin and add an 'E' at the end of the standardized SMILES to inform the sequence's ending, just like a director that commands the generator to grow fragments or to end the activity. The unified and modified sequence was then encoded using embedding method. The dictionary contained 39 words, covering the common symbols in the SMILES of organic molecules. Four numbers were used to represent special symbols, including 0 for 'pad', 1 for 'grow' $(\mathrm{G}), 2$ for 'end' (E) and 39 for 'unused symbols'. Symbols containing two letters like 'Br' and ' $\mathrm{Cl}$ ' were counted as single words. The overall encoded length for each fragment was set to 102. Fragments 
with longer length were abandoned. The dictionary can be seen as follows:

$$
\begin{gathered}
\text { \{0: 'pad', 1: 'G', 2: 'E', 3: '2', 4: 'F', 5: 'Cl', 6: 'N', 7: '[', 8: '6', 9: 'O', } \\
\text { 10: 'c', 11: ']', 12: '\#', 13: '=', 14: '3', 15: ')', 16: '4', 17: '-', 18: 'n',19: 'o', } \\
\text { 20: '5', 21: 'H', 22: '(', 23: 'C',24: '1', 25: 'S', 26: 's', 27: 'Br',28: '@',29:'+', } \\
\text { 30:'/', 31:'\', 32:'I', 33:'P', 34:'7', 35:'8', 36:'9', 37: 'X', 38:'Y', 39:'unused'\} }
\end{gathered}
$$

The graphs of core fragments utilized edges and nodes to indicate atoms and bonds separately. Each node was annotated by a total of 13 bits, standing for the presence of 'C', 'N', 'O', 'S', 'c', 'n', 'o', 's', 'H', 'F', 'I', 'Cl' and 'Br' atom respectively. The maximum number of nodes for each graph was confined to 60 . There were four edge types, accounting for single, double, triple and aromatic bond respectively. Another important property for an edge is the direction, determined by the index of its begin node and end node. The edge type together with edge direction can help create the adjacency matrix which describes the communication between nodes and facilitates the process of message traversing in the later stage. Examples and detailed illustration can be found in the original paper of GGNN ${ }^{12}$, which thoroughly explained how to represent the edge direction.

Grow Model Pretraining. Model was pretrained before the inference of reinforcement learning, which is based on an encoder-decoder network, with two input (core fragment and source fragment). The encoder composes of two parts: layers for encoding the sequence information of source fragments and layers for encoding the graph information of core fragments. The former is made up by an embedding layer with an embedding size of 39 and a GRU layer with a hidden size of 128 , which generate the embedding data and a context. The later is an implementation of the GGNN from YujiaLi and RichardZemel ${ }^{12}$. The structures of the decoder referred to that of MOSES on GitHub (https://github.com/molecularsets/moses), which is formed by three stacked GRU layers with the hidden size of 512 for each layer. Dropout rate was set to 0.2 . The embedding data and context $\mathbf{C}$ was combined as the input of the decoder, while the overall context was used to initialize the hidden state of layers. Adam optimizer with 
different learning rate $(0.0005,0.001,0.005,0.01)$ was employed to train the network. Different batch size was explored $(64,128,256)$.

Choosing Suitable Fragment Pair. Before the trained generator starts to grow, the user has to decide the fragment pair first. Considering chemists usually do not desire many variations in their designed structures, we aimed to select the best fragment pair that can be used to generate more non-blockers while keep the part of original structure (core fragment) as big as possible. Here we adopted the idea of NSGA-II to facilitate the selection. Compounds were broken into fragment pairs as above mentioned. The generator grew 500 structures for each fragment pair, which were then put into our hERG model. The fraction of predicted non-blockers for generated structures was calculated and defined as Object One for the non-dominated rank. The Object Two was defined as difference in molecule weight between core fragment and original compound. The top ranked fragment pairs were kept and finally used to grow molecules.

Comparison with Medicinal Chemists' Optimization in Previous Cases. Out of the above mentioned seven optimization cases as shown in Table 2, five cases have clear underlying optimization strategy (Case 1 5). To assess whether the optimization direction by our model was in accordance with the initial intention of the chemists, the molecules produced by our model were compared with the optimized compounds by chemists from the aspect of MolLogP, pKa, NROT and NAroR. In addition, we performed molecular docking using Schrodinger 2009 package ${ }^{19-20}$ to examine the docking conformation and docking score of optimized molecules in comparison with chemists' optimization.

We employed reinforcement learning to escalate the fraction of non-blockers in generated molecules. However, in the beginning of reinforcement learning, expert training on source fragments was first conducted to raise the similarity of target fragments to source fragments. Then, the Policy Gradient ${ }^{7}$ was applied to optimize the parameter of the network. The reward function for each generated molecule is defined 
as the non-blocker probability multiplied by the similarity of the target fragment to the source fragment (we brief it as 'similarity'). However, it is influenced by many other factors including physiochemical properties such as the number of hydrogen acceptors and donors (HBA and HBD), the number of rotatable bonds (NROT) and the fraction of nitrogen and oxygen atoms in heavy atoms (FNO), the existence of pan-assay interference compounds alerts, toxic alerts and other self-defined undesired substructures (Alerts). The SMILES arbitrary target specification (SMARTS) ${ }^{21}$ of Alerts can be seen in supporting information. Parameters for target fragments were set as follows: HBA and HBD should no more than 3; NROT should no more than 5; Similarity should be no less than 0.1 (case 4 was set to 0.3 ). Parameters for whole generated structures were set as: HBD should be no more than 5 according to rule of five; NROT should be no more than 8; FNO should be no more than 0.4 according to our previous work ${ }^{22}$; structures should not contain any alerts. Any violations of these rules will force the reward to be 0 regardless of hERG prediction results. Another manipulation is that if the similarity is higher than 0.5 , then similarity value is changed to 1 . This means that as long as the similarity reach a certain threshold, the reward will only depend on the non-blocker probability. This guarantees the model to explore some new fragments while keep a certain degree of similarity. Learning rate for reinforcement learning was set as 0.0001 . Optimization batch was 32 , and the gamma factor was set to 0.97 . The network was trained until the fraction of non-blockers for the molecule batch reached the plateau.

\section{Result and discussion}

\section{Constructing hERG Classification Model}

Feature selection. It is important to define an index that measures whether the genetic algorithm has found out the best solution (or combination) of features. In this article, we use dominance ratio, which is defined as the ratio of new individuals dominating the previous individuals to all dominating relationships. For instance, given a parental generation $\mathrm{t}-1$ and offspring generation $\mathrm{t}$, one can calculate the dominance level by 
merging the individuals of two generations. The number of times that individuals from generation $t$ rank higher than that from generation $t-1$ is counted as $\mathrm{N}_{\mathrm{t}}$ and the reverse is counted as $\mathrm{N}_{\mathrm{t}-1}$. The dominance ratio is $\mathrm{N}_{t}$ divided by the sum of $\mathrm{N}_{t}$ and $\mathrm{N}_{\mathrm{t}-1}$, which ranges from 0 to 1 . Higher ratio means the new generation can give better results than its parental generation, which suggests the evolution has not stopped and is possible to continue. Ratio of 0.5 indicates the old and the new generation ranks equally in terms of dominance level, and it is likely that evolution has reached a plateau. Ratio lower than 0.5 means the young generation is worse than the old. Figure 2 shows the variation of dominance ratio in the 100 iterations of NSGA-II. As it suggests, after the $45_{\text {th }}$ generation, the dominance ratio almost keep constant at 0.5 , implying the population has evolved to the optimal state. On the other hand, in the optimal state where new generation are basically equally competent as the previous generation, the slight advantage of one or several individuals in new population may drive the ratio to soar. For example, in extreme cases, if $\mathrm{N}_{\mathrm{t}}$ is 1 while $\mathrm{N}_{\mathrm{t}-1}$ is only 0 , the dominance ratio will be as high as 1.0. Thus, it is reasonable that the dominance ratio fluctuates between 0.5 to 1 in the balance stage.

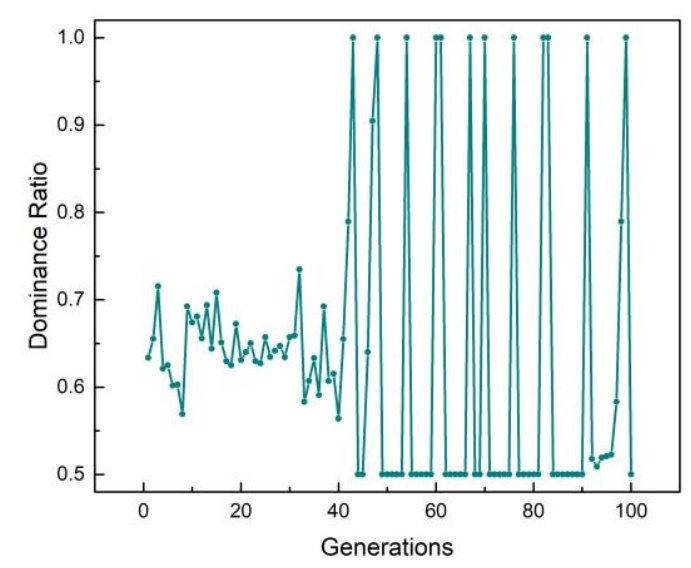

Figure 2. The Variation of Dominance Ratio in 100 Generations

Model Result. The result of eight methods for hERG classification can be seen in Table 3. In terms of the training set, six approaches including SVM, RF, ERF, AdaBoost, XGB and lightGBM show comparable 10-fold result, with AUC ranging from 0.944 to 0.951 
and ACC ranging from 0.884 to 0.889 . Their Kappa (0.744-0.757), MCC (0.747-0.758) and F1 (0.909-0.914) are also close to each other. ERF shows the highest ACC (0.889), Kappa (0.757), MCC (0.758) and second highest AUC (0.949) among these methods, indicating the best overall performance on training set. SVM and lightGBM have equal AUC (0.944), second largest Kappa and MCC (ranging from 0.752 to 0.755 ). The AUC of RF, Adaboost and XGB are marginally higher than ERF, SVM and LightGBM, but their ACC, Kappa and MCC are slightly lower than the three, from which we can conclude that difference of comprehensive performance for the six methods is quite small in terms of training set. On the other hand, SVM presents best balanced predicting ability on both blockers and non-blockers out of these methods, with a highest BAC of 0.88, which can also be demonstrated by its high sensitivity (0.901) and high specificity (0.858). By contrast, the obvious gap (about 0.159 ) between sensitivity and specificity can be observed in RF and AdaBoost, indicating their bias towards blockers when classifying molecules, probably caused by the unbalanced dataset (blockers are about 1.7 times as many as non-blockers). The bias is reduced in three methods (ERF, XGB and lightGBM), the specificity for which are all above 0.81 and the BAC for which are all above 0.871 . Compared with the two newly developed boosting methods XGB and lightGBM, GBDT seems to be weaker. It has smallest AUC (0.932), smallest BAC (0.854) and lower ACC (0.872), Kappa (0.72) and MCC (0.721), indicating relatively poor overall performance. Similarly, DNN shows the worst performance compared with other methods, with a second least AUC of about 0.934 and the smallest value in terms of ACC (0.858), Kappa (0.699), MCC (0.703), BAC (0.858), and F1(0.884). However, its predicting ability on blockers and non-blockers is quite balanced, with the sensitivity of 0.856 and specificity of 0.86 . 
Table 3. Result of Eight Classification Methods on Different Datasets

\begin{tabular}{|c|c|c|c|c|c|c|c|c|c|}
\hline \multicolumn{10}{|c|}{ Performance on Training Set $(c v=10)$} \\
\hline Method & AUC & $\mathrm{ACC}$ & Kappa & MCC & BAC & F1 & Precision & $\begin{array}{c}\text { Recall } \\
\text { (Sensitivity) }\end{array}$ & Specificity \\
\hline SVM & 0.944 & 0.886 & 0.755 & 0.755 & 0.88 & 0.909 & 0.917 & 0.901 & 0.858 \\
\hline $\mathbf{R F}$ & 0.951 & 0.884 & 0.744 & 0.747 & 0.863 & 0.912 & 0.883 & 0.942 & 0.783 \\
\hline ERF & 0.949 & 0.889 & 0.757 & 0.758 & 0.873 & 0.914 & 0.898 & 0.931 & 0.816 \\
\hline AdaBoost & 0.947 & 0.885 & 0.745 & 0.748 & 0.863 & 0.912 & 0.884 & 0.942 & 0.784 \\
\hline GBDT & 0.932 & 0.872 & 0.72 & 0.721 & 0.854 & 0.901 & 0.884 & 0.92 & 0.789 \\
\hline XGB & 0.945 & 0.884 & 0.747 & 0.747 & 0.871 & 0.909 & 0.901 & 0.918 & 0.824 \\
\hline LightGBM & 0.944 & 0.886 & 0.752 & 0.753 & 0.872 & 0.912 & 0.899 & 0.925 & 0.819 \\
\hline DNN & 0.934 & 0.858 & 0.699 & 0.703 & 0.858 & 0.884 & 0.915 & 0.856 & 0.86 \\
\hline \multicolumn{10}{|c|}{ Performance on Test Set } \\
\hline Method & AUC & $\mathrm{ACC}$ & Kappa & MCC & BAC & F1 & Precision & $\begin{array}{c}\text { Recall } \\
\text { (Sensitivity) }\end{array}$ & Specificity \\
\hline SVM & 0.949 & 0.889 & 0.763 & 0.764 & 0.886 & 0.911 & 0.924 & 0.898 & 0.874 \\
\hline $\mathbf{R F}$ & 0.953 & 0.897 & 0.774 & 0.778 & 0.877 & 0.922 & 0.891 & 0.955 & 0.799 \\
\hline ERF & 0.947 & 0.895 & 0.77 & 0.772 & 0.879 & 0.919 & 0.899 & 0.939 & 0.819 \\
\hline AdaBoost & 0.944 & 0.891 & 0.76 & 0.763 & 0.871 & 0.917 & 0.888 & 0.947 & 0.794 \\
\hline GBDT & 0.932 & 0.877 & 0.731 & 0.732 & 0.86 & 0.905 & 0.886 & 0.924 & 0.795 \\
\hline XGB & 0.95 & 0.891 & 0.765 & 0.765 & 0.88 & 0.915 & 0.907 & 0.922 & 0.838 \\
\hline LightGBM & 0.947 & 0.889 & 0.759 & 0.759 & 0.876 & 0.913 & 0.902 & 0.924 & 0.828 \\
\hline DNN & 0.942 & 0.873 & 0.726 & 0.726 & 0.862 & 0.9 & 0.896 & 0.903 & 0.821 \\
\hline \multicolumn{10}{|c|}{ Performance on External Set } \\
\hline Method & AUC & $\mathrm{ACC}$ & Kappa & $\mathrm{MCC}$ & BAC & F1 & Precision & $\begin{array}{c}\text { Recall } \\
\text { (Sensitivity) }\end{array}$ & Specificity \\
\hline SVM & 0.908 & 0.845 & 0.69 & 0.698 & 0.852 & 0.853 & 0.919 & 0.796 & 0.908 \\
\hline RF & 0.943 & 0.869 & 0.73 & 0.732 & 0.862 & 0.887 & 0.866 & 0.908 & 0.817 \\
\hline ERF & 0.935 & 0.869 & 0.732 & 0.732 & 0.865 & 0.885 & 0.876 & 0.894 & 0.835 \\
\hline AdaBoost & 0.929 & 0.837 & 0.664 & 0.666 & 0.828 & 0.861 & 0.83 & 0.894 & 0.761 \\
\hline GBDT & 0.906 & 0.825 & 0.643 & 0.643 & 0.822 & 0.845 & 0.845 & 0.845 & 0.798 \\
\hline XGB & 0.928 & 0.861 & 0.717 & 0.717 & 0.86 & 0.875 & 0.885 & 0.866 & 0.853 \\
\hline LightGBM & 0.926 & 0.869 & 0.734 & 0.734 & 0.869 & 0.882 & 0.898 & 0.866 & 0.872 \\
\hline DNN & 0.911 & 0.853 & 0.702 & 0.703 & 0.853 & 0.866 & 0.889 & 0.845 & 0.862 \\
\hline
\end{tabular}

As for the test set, SVM, RF, ERF, AdaBoost, XGB and lightGBM continue to show different degree of superiority over GBDT and DNN in terms of the comprehensive indexes AUC, ACC, BAC, Kappa and $\mathrm{MCC}$, despite that DNN witnessed an improvement of AUC (0.942) on test set. AUC for the six methods remains in the range 
from 0.944 to 0.953 , compared with 0.932 of GBDT and 0.942 of DNN. ACC cluster between 0.889 and 0.897 , compared with 0.877 of GBDT and 0.873 of DNN. Kappa and MCC follow a similar pattern. Among the six superior methods, RF and ERF demonstrates their best overall predicting competence on test set, with the ACC of 0.897 and 0.895 , the Kappa of 0.774 and 0.77 and the MCC of 0.778 and 0.772 respectively. The performance of SVM, AdaBoost, XGB and lightGBM are close to each other in terms of AUC, ACC, Kappa and MCC, despite that Kappa and MCC for lightGBM are slightly lower (0.759). However, SVM shows the best BAC of 0.886 and its specificity (0.874) are approximate to sensitivity (0.898), suggesting its good applicability for both blockers and non-blockers in test set.

Different from the result of training set and test set where the gap between eight methods is relatively small, their generalization ability on clinical external set varies from method to method. As shown in Table 3, the ACC for RF, ERF and lightGBM can reach more than 0.869 , and their Kappa/MCC value are all above 0.73 , indicating their good generalization ability. In addition, lightGBM shows the best balanced predicting ability on external set, with the highest BAC of 0.869 and close sensitivity $(0.866)$ and specificity (0.872). There is a significant decline in the predicting performance of SVM and AdaBoost, with Kappa value reducing to only 0.69 and 0.664 respectively. This result reveals that SVM and AdaBoost may have poor generalization ability. GBDT still takes the last place in terms of AUC, ACC, Kappa and MCC, which obviously should not be considered to be selected. DNN maintains its predicting competence on the same level in the three datasets (Kappa value remains around 0.70); however, it is still weaker than RF, ERF and lightGBM in this study.

According to Table 3, RF, ERF and lightGBM show favorable performance on all the three datasets. SVM generates a desirable result on training set and test set, but its generalization ability on external set is relatively poor. The difference is further illustrated in Figure S1, the total number of wrongly classified structures by SVM (39) is larger than that of the other three models (33), revealing SVM has limited 
generalization capability. Table 4 illustrates the four models' BAC on different external subsets scaled by similarity to the training set. All methods obtain excellent result when the similarity is less than 0.7 , with BAC greater than 0.8 . However, the difference is enlarged when the threshold of similarity is limited to 0.3 . ERF remains best classifying competence (0.789). RF and LightGBM can also reach a good BAC at this threshold (more than 0.73), demonstrating their potential in classifying new structures. By contrast, BAC of SVM experiences a significant drop to 0.661 at 0.3 , further demonstrating its poor generalization ability.

Table 4. Balanced Accuracy on Different External Subsets Scaled by Similarity

\begin{tabular}{cccccc}
\hline Similarity $^{\mathbf{a}}$ & Count & RF & ERF & LightGBM & SVM \\
\hline$\geq 0.8$ & 77 & 0.974 & 0.974 & 0.961 & 0.951 \\
$<0.8$ & 174 & 0.822 & 0.822 & 0.828 & 0.808 \\
$<\mathbf{0 . 7}$ & $\mathbf{1 2 6}$ & $\mathbf{0 . 8 1 0}$ & $\mathbf{0 . 8 2 5}$ & $\mathbf{0 . 8 1 7}$ & $\mathbf{0 . 8 0 1}$ \\
$<0.6$ & 81 & 0.765 & 0.790 & 0.753 & 0.760 \\
$<0.5$ & 64 & 0.734 & 0.781 & 0.734 & 0.738 \\
$<0.4$ & 46 & 0.761 & 0.783 & 0.739 & 0.723 \\
$<\mathbf{0 . 3}$ & $\mathbf{1 9}$ & $\mathbf{0 . 7 3 7}$ & $\mathbf{0 . 7 8 9}$ & $\mathbf{0 . 7 3 7}$ & $\mathbf{0 . 6 6 1}$ \\
\hline
\end{tabular}

a Compounds' similarity to the training set.

Validation on Optimization Cases. RF, ERF and LightGBM is further evaluated on seven optimization cases to investigate their sensitivity to subtle structural difference, which can be seen in Table 5. The before-optimization compounds (hERG channel blockers) are labeled as 1, and after-optimization compounds (hERG channel nonblockers) are labeled as 0 . Only when the models correctly predict both the before- and after- structures can we name it a successful case. LightGBM classifies successfully five cases in total, dominating the first place, followed by only 4 cases of RF and 3 cases of ERF. Besides, the probability predicted by LightGBM shows a downward trend from 'before' to 'after', even in the two wrongly predicted cases of LightGBM (Case 2 and 4). In comparison, a false trend in Case 7 can be observed for ERF. From Table 2, we can know that Case 7 is relatively difficult to predict, as the only distinction between 7a and $\mathbf{7 b}$ is the position difference of the methylsufonyl group. Only lightGBM 
successfully distinguishes the two structures, which suggests it may be the best model to recognize small structural variations.

Table 5. Predicting Result of Literature Compounds

\begin{tabular}{|c|c|c|c|c|c|c|c|c|}
\hline \multicolumn{3}{|c|}{ Literature Compounds } & \multicolumn{2}{|c|}{ RF } & \multicolumn{2}{|c|}{ ERF } & \multicolumn{2}{|c|}{ LightGBM } \\
\hline Case & Compound ID & True Label & Pred Labe & el PredProb ${ }^{\mathrm{a}}$ & Pred Label & PredProb & Pred Label & PredProb \\
\hline \multirow{2}{*}{1} & $1 \mathrm{a}$ & 1 & 1 & 0.727 & 1 & 0.785 & 1 & 0.957 \\
\hline & $1 b$ & 0 & 0 & 0.415 & 0 & 0.274 & 0 & 0.268 \\
\hline \multirow{2}{*}{2} & $2 \mathrm{a}$ & 1 & 1 & 0.871 & 1 & 0.915 & 1 & 1.000 \\
\hline & $2 b$ & 0 & 1 & 0.678 & 1 & 0.769 & 1 & 0.993 \\
\hline \multirow{2}{*}{3} & $3 a$ & 1 & 1 & 0.536 & 1 & 0.508 & 1 & 0.675 \\
\hline & $3 b$ & 0 & 0 & 0.393 & 0 & 0.431 & 0 & 0.001 \\
\hline \multirow{2}{*}{4} & $4 a$ & 1 & 1 & 0.796 & 1 & 0.710 & 1 & 0.997 \\
\hline & $4 b$ & 0 & 1 & 0.660 & 1 & 0.615 & 1 & 0.988 \\
\hline \multirow{2}{*}{5} & $5 a$ & 1 & 1 & 0.513 & 0 & 0.356 & 1 & 0.885 \\
\hline & $5 b$ & 0 & 0 & 0.347 & 0 & 0.385 & 0 & 0.006 \\
\hline \multirow{2}{*}{6} & $6 a$ & 1 & 1 & 0.824 & 1 & 0.777 & 1 & 0.997 \\
\hline & $6 b$ & 0 & 0 & 0.472 & 0 & 0.357 & 0 & 0.318 \\
\hline \multirow{3}{*}{7} & $7 \mathrm{a}$ & 1 & 1 & 0.633 & 1 & 0.723 & 1 & 0.857 \\
\hline & $7 b$ & 0 & 1 & 0.625 & 1 & 0.731 & 0 & 0.332 \\
\hline & \multicolumn{2}{|c|}{ Correct Cases } & \multicolumn{2}{|r|}{4} & \multicolumn{2}{|c|}{3} & \multicolumn{2}{|c|}{5} \\
\hline
\end{tabular}

a Predicted probability of being a hERG channel blocker by a classfication model.

Compared with Other Works. Our work is compared with the previous studies in Table 6. From the table we can see, there is no unified standard on setting the thresholds for computational chemists. Thresholds include both single threshold such as $1 \mu \mathrm{M}, 10 \mu \mathrm{M}$, $50 \%$ efficacy, $50 \%$ inhibition at $10 \mu \mathrm{M}$, as well as double threshold such as $\leq 10$ $\mu \mathrm{M},>10 \mu \mathrm{M}$ ', ' $\leq 10 \mu \mathrm{M} \geq 80 \mu \mathrm{M}$ ', ' $<10 \mu \mathrm{M}>30 \mu \mathrm{M}$ '. Different thresholds may impose different influence on model performance. If the $\mathrm{IC}_{50}$ gap between the defined 'blocker' and 'non-blocker' is large, the model performance will be improved according to Chuipu Cai et $\mathrm{al}^{23}$. However, this improvement might suffer from losing some data. So we should take both model performance and dataset size into account. According to the table, from the perspective of model performance our lightGBM model generates a good ACC (0.889), good BAC (0.876), high AUC (0.947), high Kappa (0.759) and MCC (0.759) on test set compared with other models. Only three works shows obvious 
advantages over us with ACC greater than 0.9, which are studies from Chuipu Cai et al. ${ }^{23}$, Li-li LIU et al. ${ }^{24}$ and Yiwei Wang et al. ${ }^{25}$. However, Chuipu Cai and co-workers chose a double threshold with large gap ' $\leq 10 \mu \mathrm{M} \geq 80 \mu \mathrm{M}$ ', with a dataset of only 4,447 data points, in comparison with our 11,599 data points. Similarly, although Li-li LIU et al. ${ }^{24}$ and Yiwei Wang et al. ${ }^{25}$ employed almost the same thresholds as us and obtained a quite good result, their data size is only 2,644 , which is only about $23 \%$ of ours.

Table 6. Comparison of Our hERG Classfication Model with Previous work.

\begin{tabular}{|c|c|c|c|c|c|c|}
\hline Year & Author & Method & Descriptors & Threshold & Data Size & Performance \\
\hline 2015 & $\begin{array}{c}\text { Rodolpho C. Braga } \\
\text { et al. }{ }^{26}\end{array}$ & SVM & $\begin{array}{l}\text { Fingerprints and } \\
\text { CDK descriptors }\end{array}$ & $1 \mu \mathrm{M}, 10 \mu \mathrm{M}$ & 5,984 & $\begin{array}{l}\mathrm{ACC}_{\text {train }}(\mathrm{CV}=5)=0.88 \\
\mathrm{AUC}_{\text {train }}(\mathrm{CV}=5)=0.88 \\
\mathrm{MCC}_{\text {train }}(\mathrm{CV}=5)=0.56\end{array}$ \\
\hline 2016 & $\begin{array}{c}\text { Remigijus } \\
\text { Didziapetris et al. }{ }^{27}\end{array}$ & GBM & $\begin{array}{l}\text { Physicochemical } \\
\text { and topological } \\
\text { parameters }\end{array}$ & $\leq 10 \mu \mathrm{M}>10 \mu \mathrm{M}$ & 6,124 & $\begin{array}{l}\mathrm{ACC}_{\text {test }}=0.72-0.75 \\
\mathrm{AUC}_{\text {test }}=0.80-0.83 \\
\mathrm{MCC}_{\text {test }}=0.42-0.50\end{array}$ \\
\hline 2017 & $\begin{array}{c}\text { Hongmao Sun et } \\
\text { al. }^{28}\end{array}$ & SVM & $\begin{array}{l}\text { Atom-type-based } \\
\text { molecular descriptor }\end{array}$ & $>50 \%$ efficacy & 3,024 & $\mathrm{AUC}_{\text {train }}=0.93$ \\
\hline 2018 & $\begin{array}{c}\text { Vishal B. } \\
\text { Siramshetty et al. } .^{29}\end{array}$ & $\mathrm{NN}, \mathrm{SVM}, \mathrm{RF}$ & Fingerprints & $\leq 1 \mu \mathrm{M} \geq 10 \mu \mathrm{M}$ & 3,223 & $\begin{array}{l}\mathrm{BAC}_{\text {train }}(\mathrm{CV}=10)=0.87 \\
\mathrm{AUC}_{\text {train }}(\mathrm{CV}=10)=0.94\end{array}$ \\
\hline 2019 & Keiji Ogura et al. ${ }^{2}$ & SVM & $\begin{array}{l}\text { Fingerprint, MOE, } \\
\text { Pipeline Pilot } \\
\text { descriptors }\end{array}$ & $\begin{array}{c}10 \mu \mathrm{M}, \\
50 \% \text { inhibition at } \\
10 \mu \mathrm{M}\end{array}$ & 291,202 & $\begin{aligned} \mathrm{BAC}_{\text {test }} & =0.839 \\
\mathrm{Kappa}_{\text {test }} & =0.749\end{aligned}$ \\
\hline 2019 & $\begin{array}{c}\text { Hyang-Mi Lee et } \\
\text { al. }{ }^{30}\end{array}$ & Neural Network & $\begin{array}{l}\text { Fingerprints and } \\
\text { physicochemical } \\
\text { descriptors }\end{array}$ & $<10 \mu \mathrm{M} \geq 10 \mu \mathrm{M}$ & 2,130 & $\begin{array}{l}\mathrm{ACC}_{\text {train }}(\mathrm{CV}=10)=0.910 \\
\mathrm{AUC}_{\text {train }}(\mathrm{CV}=10)=0.764 \\
\mathrm{MCC}_{\text {train }}(\mathrm{CV}=10)=0.368\end{array}$ \\
\hline 2019 & Chuipu Cai et al. ${ }^{23}$ & $\begin{array}{c}\text { DNN,GCNN,NB, } \\
\text { SVM,RF }\end{array}$ & Mol2vec,MOE(2D) & $\leq 10 \mu \mathrm{M} \geq 80 \mu \mathrm{M}$ & 4,447 & $\begin{array}{l}\mathrm{ACC}_{\text {test }}=0.925 \\
\mathrm{AUC}_{\text {test }}=0.967\end{array}$ \\
\hline 2020 & Hyunho Kim et al. ${ }^{31}$ & $\begin{array}{c}\text { Self-attention } \\
\text { network }\end{array}$ & Fingerprints & $\leq 10 \mu \mathrm{M},>10 \mu \mathrm{M}$ & 11,860 & $\mathrm{AUC}_{\text {test }}=0.89$ \\
\hline 2020 & $\begin{array}{c}\text { Jae Yong Ryu et } \\
\mathrm{al}^{32}\end{array}$ & DNN,GCN & $\begin{array}{c}\text { Moredred } \\
\text { Descriptors,Graph }\end{array}$ & $<10 \mu \mathrm{M} \geq 10 \mu \mathrm{M}$ & 14,440 & $\begin{array}{l}\mathrm{ACC}_{\text {test }}=0.812 \\
\mathrm{MCC}_{\text {test }}=0.641\end{array}$ \\
\hline 2010 & $\begin{array}{c}\text { Munikumar R. } \\
\text { Doddareddy et al. }{ }^{15}\end{array}$ & SVM,LDA & Fingerprints & $<10 \mu \mathrm{M}>30 \mu \mathrm{M}$ & 2,644 & $\begin{array}{l}\mathrm{ACC}_{\text {train }}(\mathrm{CV}=5)=0.93 \\
\mathrm{MCC}_{\text {train }}(\mathrm{CV}=5)=0.70\end{array}$ \\
\hline 2014 & Li-li LIU et al. ${ }^{24}$ & NBC & $\begin{array}{c}\text { Fingerprints and } 4 \\
\text { molecular properties }\end{array}$ & $<10 \mu \mathrm{M}>30 \mu \mathrm{M}$ & 2,644 & $\mathrm{ACC}_{\text {test }}=0.91$ \\
\hline 2020 & Yiwei Wang et al. ${ }^{25}$ & Capsule Networks & $\begin{array}{c}\text { Fingerprints, } 13 \\
\text { molecular properties }\end{array}$ & $<10 \mu \mathrm{M}>30 \mu \mathrm{M}$ & 2,644 & $\begin{array}{l}\mathrm{ACC}_{\text {test }}=0.918 \\
\mathrm{AUC}_{\text {test }}=0.940 \\
\mathrm{MCC}_{\text {test }}=0.835\end{array}$ \\
\hline
\end{tabular}




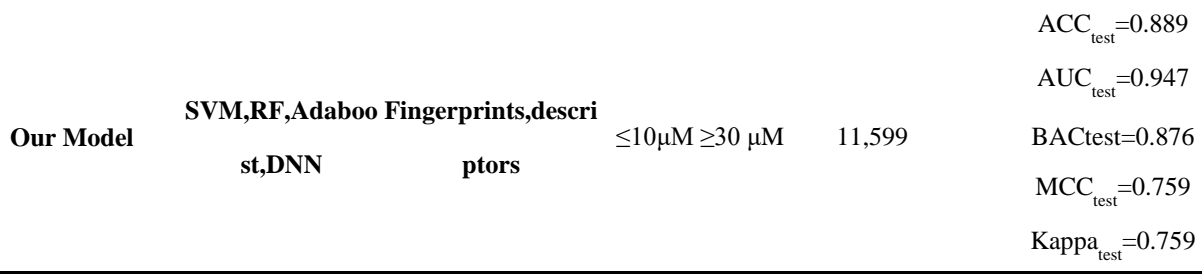

\section{Fragment Grow Network}

Grow Model Construction. Different GGNN-RNN models parameterized by different batch size and learning rate were constructed. 1000 molecules were generated on core fragment ' $\left[{ }^{*}\right] \mathrm{C} 1=\mathrm{CC}=\mathrm{C} 2 \mathrm{C}(\mathrm{C}=\mathrm{CN} 2)=\mathrm{C} 1$ ' and source fragment ${ }^{*}$ Oc1ncnc2c1CCNC2' for each model. The fraction of valid structures is shown in Figure 3. As it suggests, model performance is very sensitive to the change of learning rate, varying largely from 0.001 to 0.96 . The best value is 0.001 , at which all models can reach equal to or more than 0.9 validity, regardless of the batch size. In comparison, variation in batch size seems to matter less, but it still generates a difference of 0.33 at learning rate 0.0005 . Batch size 128 and learning rate 0.001 were chosen to build our model, the validity of which is 0.96 .

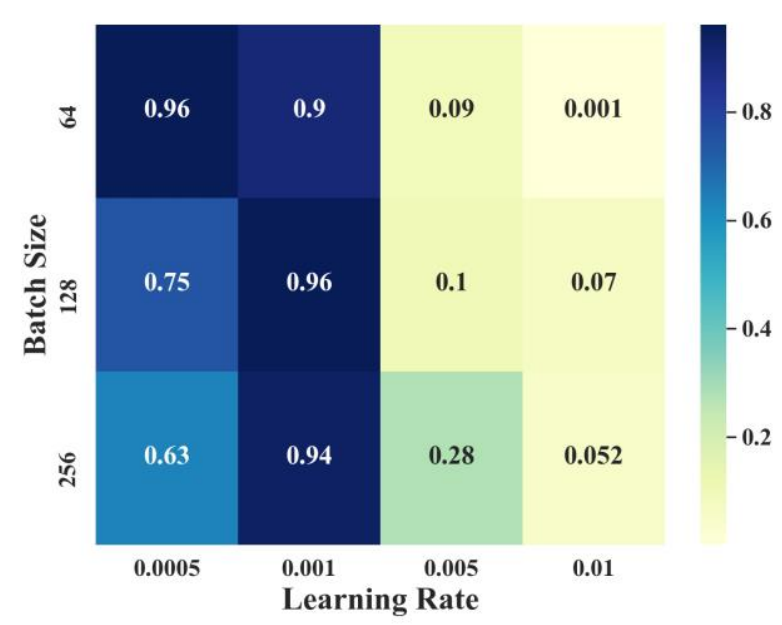

Figure 3. Fraction of Valid Structures Generated by Different GGNN-RNN Models

In order to vertify the addition of GGNN can facilitate the molecule grow process, we compare our GGNN-RNN model with the raw RNN model, which replaces the GGNN encoder with a single GRU layer to encode the context information of the core fragment. 
1000 molecules were generated based on core fragment ' $[*] \mathrm{C} 1=\mathrm{CC}=\mathrm{C} 2 \mathrm{C}(\mathrm{C}=\mathrm{CN} 2)=\mathrm{C} 1$ ' and source fragment '*Oc1ncnc2c1CCNC2'. The proportion of valid SMILES produced by raw GNN-RNN is much higher than the raw RNN model, which is only 0.792. Moreover, it has lower average Synthetic Accessibility Score (SA Score), which is 2.316, suggesting the structures it generates may be more easier to synthesize compared with raw RNN model (2.745). The difference of SA Score is significant at 95\% confidence level. The reason for these results may be that the node level GGNN can extract the information of a particular growing site on core fragments, instead of broad information that expresses the whole fragments. Therefore, the network can gather more surrounding information of the starting point before growing, which may make the link between core fragments and target fragments to be more authentic (or more valid). Thus the fraction of valid structures and the synthetic accessibility will increase.

Choosing Suitable Fragment Pair. Before reinforcement learning starts, it is necessary to decide which fragment pair to feed into the model, as cutting on different bonds will generate different fragment pairs. In order to search for the most suitable fragment with less hERG risk as well as reserve the original structure as much as possible, fragments are put into the pretrained GGNN-RNN model and the fraction of non-blockers in the output is recorded as a reference for selection. The idea of NSGA-II was employed to find the best solution. As we can see from Figure 4, this method successfully offers suitable core fragments which are in aggreement with chemists' final decisions in four cases. In Case 1, five core fragments are suggested by NSGA-II sorting. Model that grows on core fragment $\mathbf{1 - 3}$ can generate $89 \%$ non-blockers in valid SMILES, but 178.66 Da of the molecule has to be cut down. By contrast, fragment 1-4 only abandons 84.08 Da molecule weight but provides $52.3 \%$ chance to obtain a non-blocker even when reinforcement learning is not performed, which is promising to be a core fragment. Drug designers in this case actually chose the fragment 1-4 as core fragment for hERG optimization, demonstrating the practicability of our technique. In Case 2, the three fragments are proposed by the model, the molecule weight (MW) difference of which 
is relatively small. Fragment $\mathbf{2 - 1}$ can provide the much higher chance $(49.6 \%)$ than $\mathbf{2}$ $3(21.9 \%)$ to generate a non-blocker according to our result, which is also chosen by chemists. Similarly, fragment 3-1 in Case 3 and 5-2 in Case 5 can give $83.8 \%$ and $82.6 \%$ chance in our calculation. Both of them are taken as core fragments by drug designers. In Case 4, no fragments are exacly the same as the choice of chemists. However, all of them reserve the triazole and piperidine parts, which may give chemists some ideas to choose the core fragment. It should be noted that circumstances vary from case to case, as drugs usually directing at a special target through special functional groups. The method cannot tell which fragment is the best one using unified standards but offer chemists with several options. Although it needs some human involvement in this step, it greatly reduces the human labor by limiting the scope of selection.

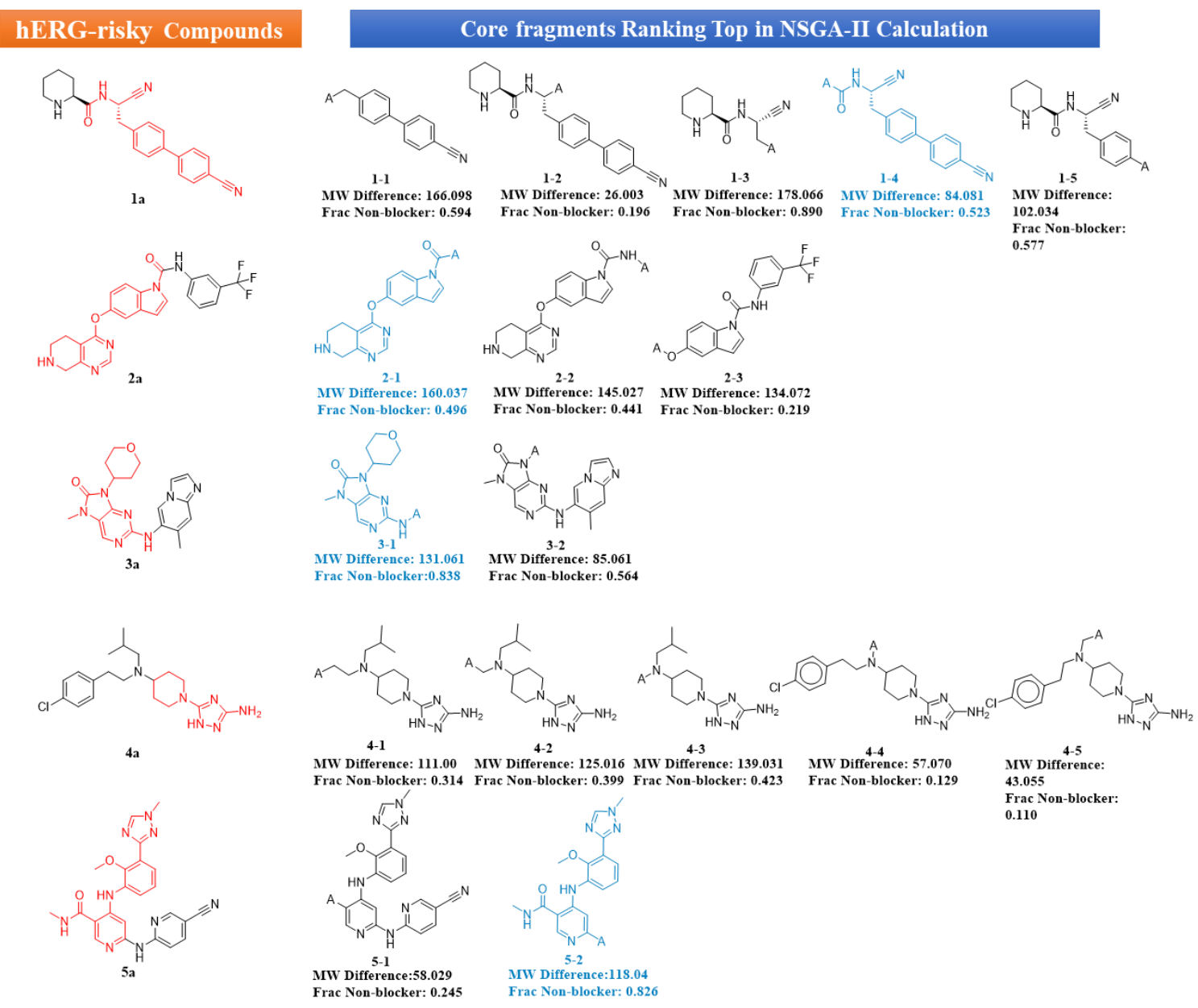

Figure 4. Comparison of Core Fragments Chosen by Chemists with Our Models' Suggestion. On the left side, original compound in each optimization case is provided 
and the kept core fragment by chemists is marked by red. On the right side, core fragments suggested by NSGA-II are displayed together with their difference in MW to the original compound and fraction of non-blockers they generated after being fed into the pretrained GGNN-RNN model.

Comparison with Medicinal Chemists' Optimization. Assuming that chemists have chosen red parts as core fragments and the corresponding rest as source fragments in Figure 4, the next step is to feed those fragments into the model. We performed about 60 140 epochs for each case to improve the hERG property. 1000 molecules were generated after the model was trained. Invaild molecules, duplicates and predicted hERG channel blockers were removed. Here we will explain the result case by case.

Case 1. 100 epochs of reinforcement learning were conducted on core fragment 1-4 and corresponding source fragment. 20 predicted non-blockers were obtained after molecule manipulaton. Their SMILES, hERG-channel-blocker probability, similarity to the original structures, docking score to the original target can be seen in Table S2. Here we display some reprensentatives in Figure 5. The main idea of the chemists in this case is to lower lipophilicity to reduce the hERG risk. As shown in Figure $\mathbf{5}$ and ClogP drops from 2.706 to 0.666 after optimization. Our grow model also suggests some structures with lower CLogP, such as 1-4-1, 1-4-2, 1-4-3 and 1-4-4. Their hERGblocker probability is $0.025,0.015,0.005$ and 0.112 respectively, which is the lowest among the proposed structures. Structures that do not show obvious decrease in CLogP such as 1-4-5 and 1-4-6 are also suggested, but their hERG-blocker probability is higher (0.37 and 0.38$)$, revealing that the trend of prediction agrees with the chemists' experience. After molecular docking, we find that 1-4-2 can adopt the most similar docking conformation with $\mathbf{1 b}$ and keep key interactions ${ }^{33}$ with the original target (Figure S2), which suggests that our model can provide reasonable optimization direction such as lowering CLogP while reserving the binding mode. 
Case 1

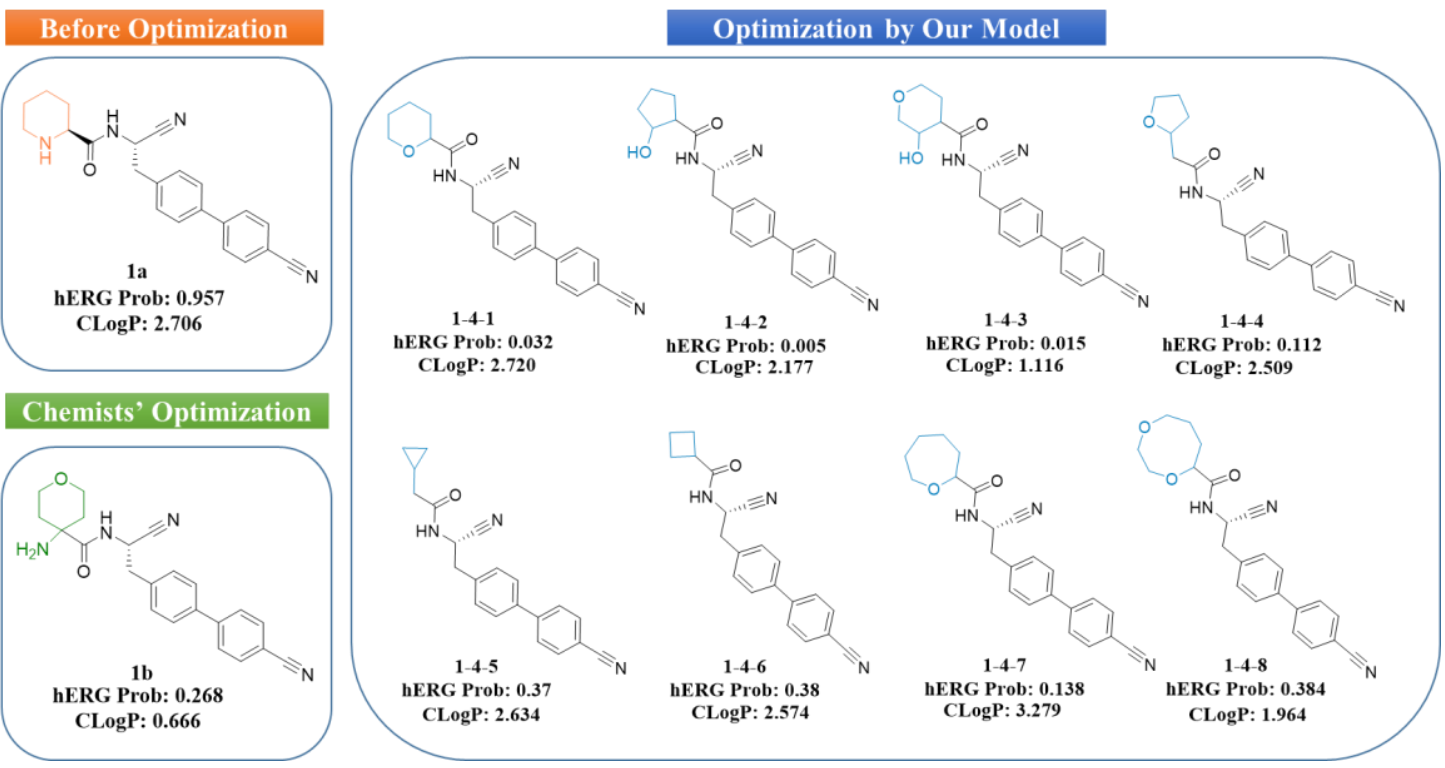

Figure 5. Examples of Optimized Structures in Case 1

Case 2. Chemists in Case 2 also adopted a 'lowering lipophilicity' policy. Therefore, CLogP is also used for analyzing the result in this case. Model was trained for 140 epochs using reinforcement learning based on core fragment 2-1 and corresponding source fragment. 16 possible non-blockers were suggested after removing duplicates. Detailed information can be seen in Table S3. As shown in Figure 6 and Table S3, the majority of structures have lower CLogP compared with the orgininal structure 2a, demonstrating that reinforcement learning drives our model to produce structures with lower CLogP. Structure 2-1-1 and 2-1-2 advice to reduce the six-membered ring to fivemembered ring and add some nitrogen to the cycle. This idea is similar to chemists' choice to some extent. Docking conformation comparison between $\mathbf{2 b}$ and 2-1-1 demonstrates our optimization may be welcomed by chemists except for the loss of small hydrophobic structures (Figure S3). 2-1-3 and 2-1-4 employ nitrogen heterocycles as substitutes for bezene ring, which may provide another possible options for chemists to consider. 2-1-5 $\sim \mathbf{2 - 1 - 8}$ recommend to replace the ring with some polar side chains or short carbon chains. Although lipophilicity and hERG-channel-blocker probability is lowered in these structures, chemists might not consider them due to great structural flexibility. 
Case 2

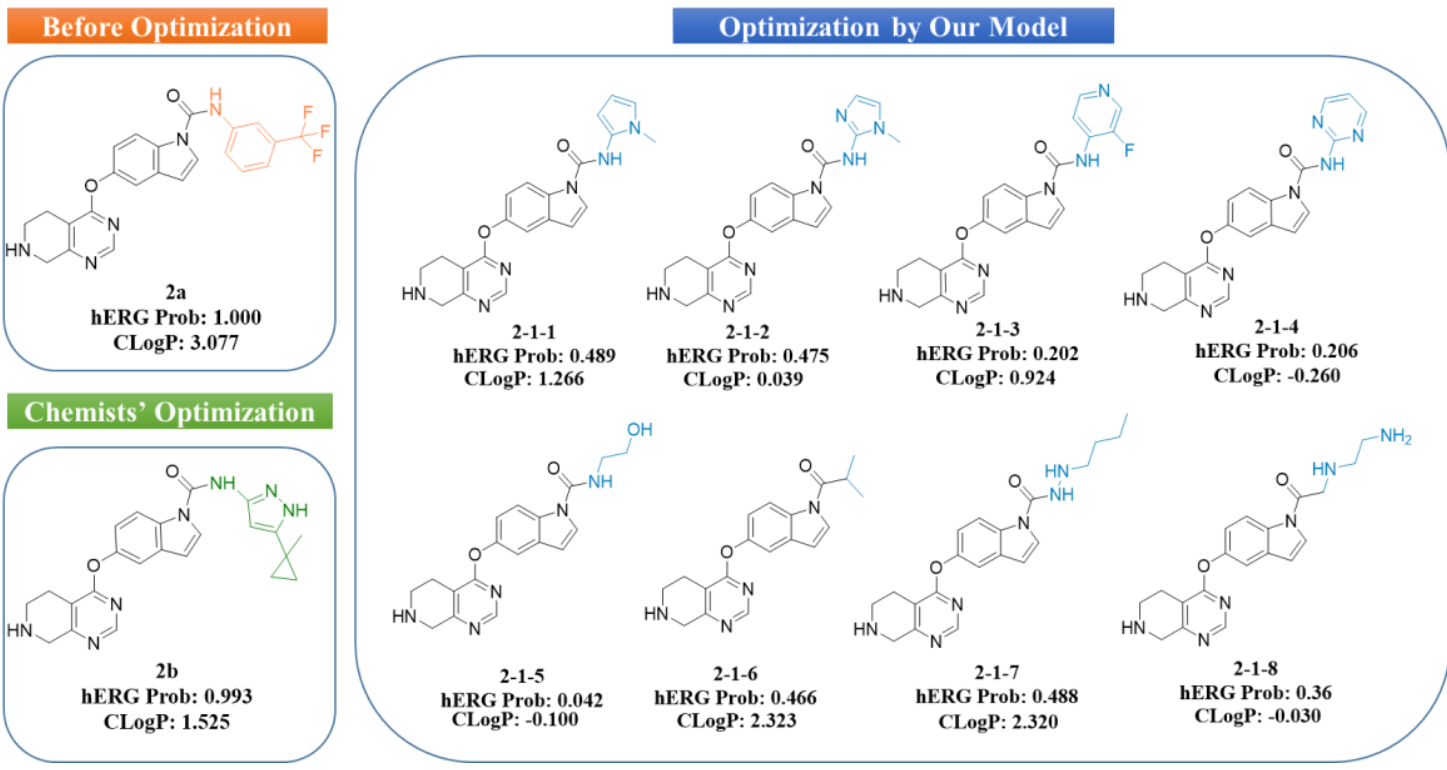

Figure 6. Examples of Optimized Structures in Case 2

Case 3. Lowering basicity is the optimization strategy employed by chemists in Case 3. Thus, we utilize pKa to anlayze the result. After 100 runs of training on core fragment 3-1 and corresponding source fragment as well as molecule clean, 33 potential nonblockers were attained. Their SMILES, pKa, hERG probability, similarity to the original structure and docking score to the original target are recorded in Table S4. Some examples can be seen in Figure 7. As shown in Figure 7 and Table S4, the pKa value for most structures suggested by our model are smaller than 3a, except for several outliers such as 3-1-8. Their hERG-channel-blocker probability is very low, nearly 0 for most structures, implying successful optimization has been achieved. Transformation by our model mainly involves the addition of nitrogen in different bicycles, which is very similar to the chemists' strategy (3b). The comparison of docking conformation between $\mathbf{3 b}$ and 3-1-1 shows that our optimization can well preserve the desired binding mode, suggesting it can provide suitable optimization direction for medicinal chemists to consider (Figure S4). 
Case 3

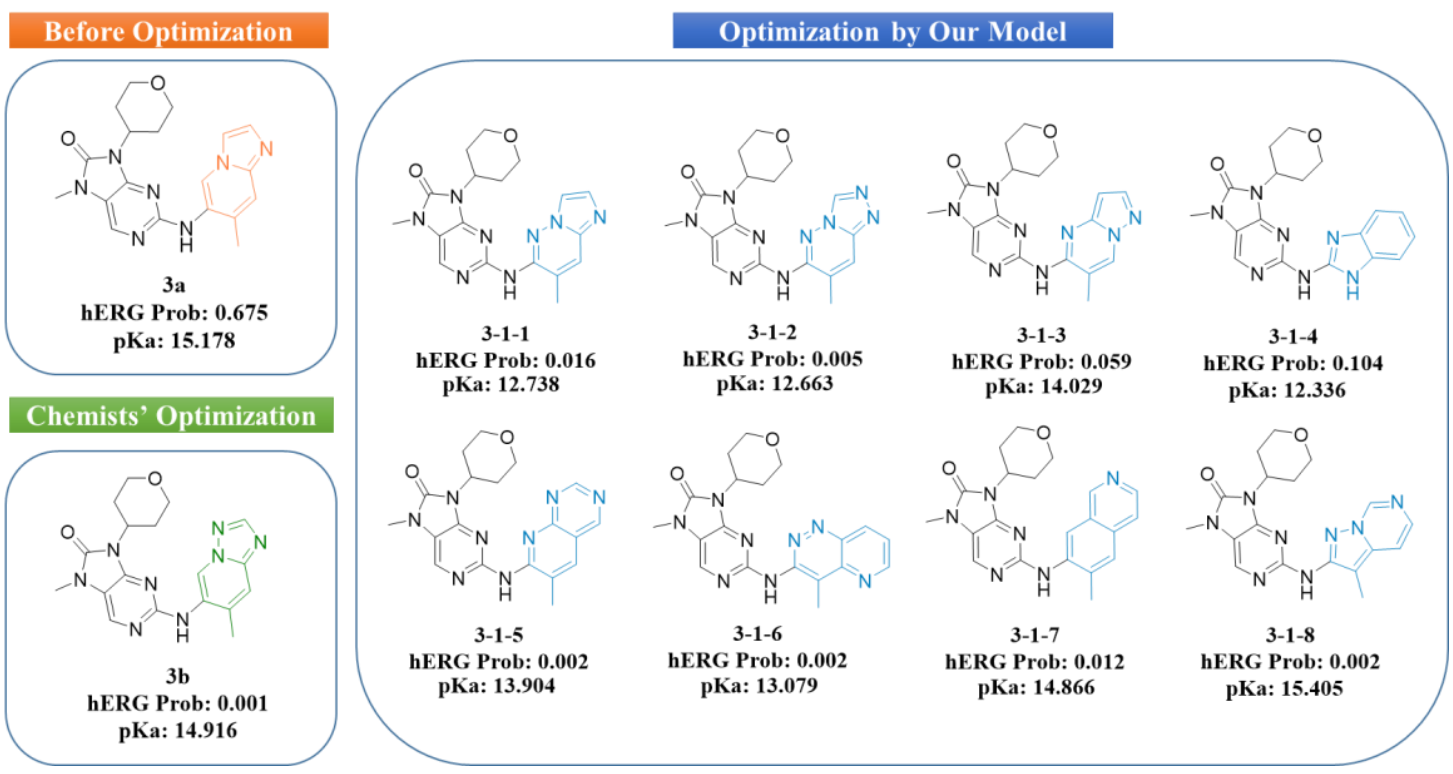

Figure 7. Examples of Optimized Structures in Case 3

Case 4. Flexible linkers allow compounds to adopt better conformations in hERG channel $^{3}$. Therefore, rigidification has been proposed a reliable approach to reduce hERG risk. In Case 4, chemists explored structure-activity relationship (SAR) of hERG ion channel by derivatives of dofetilide ${ }^{34}$, which is a sulfonamide class III antiarrhythmic agent and potassium channel blocker. So the specific target of this case is hERG potassium channel. As shown in Figure 8, Chemists restrained the conformation by forming a ring and decreasing the rotatable bonds, which greatly reduces the hERG binding affinity. In comparison, our model suggests many optimization directions. 4-0-1 4-0-4 reduce the rotatable bonds by directly cutting down rotatable linkers. 4-0-5 and 4-0-6 reduce the flexibility by using a urea and an amide as a linker respectively. 4-0-7 abandons the benzene ring instead of cutting rotatable bonds, which is likey to reduce the pi-pi interaction with Phe 656 in hERG potassium channel ${ }^{35}$. 4-0-8 is most similar to the original structure $\mathbf{4 a}$, with a long flexible linker. Its hERG probability is relatively higher $(0.315)$, suggesting that the trend of prediction is consistent with the chemists' experience. Docking scores of 70 generated structures after 100-epoch reinforcement training are shown in Table S5 (docking to the homology model of hERG potassium channel ${ }^{35}$ ). Absolute value of 
docking score for the majority of structures is smaller than chemists' optimization $\mathbf{4 b}$, indicating that the interaction between these structures and the hERG ion channel may be weaker and thus the hERG toxicity may be smaller. Case 4 shows that the decrease of NROT is not the major action taken by our model. It is reasonble because reducing NROT is not the final purpose of hERG improvement, but a technique to avoid some conformations prone to binding with hERG ion channel.

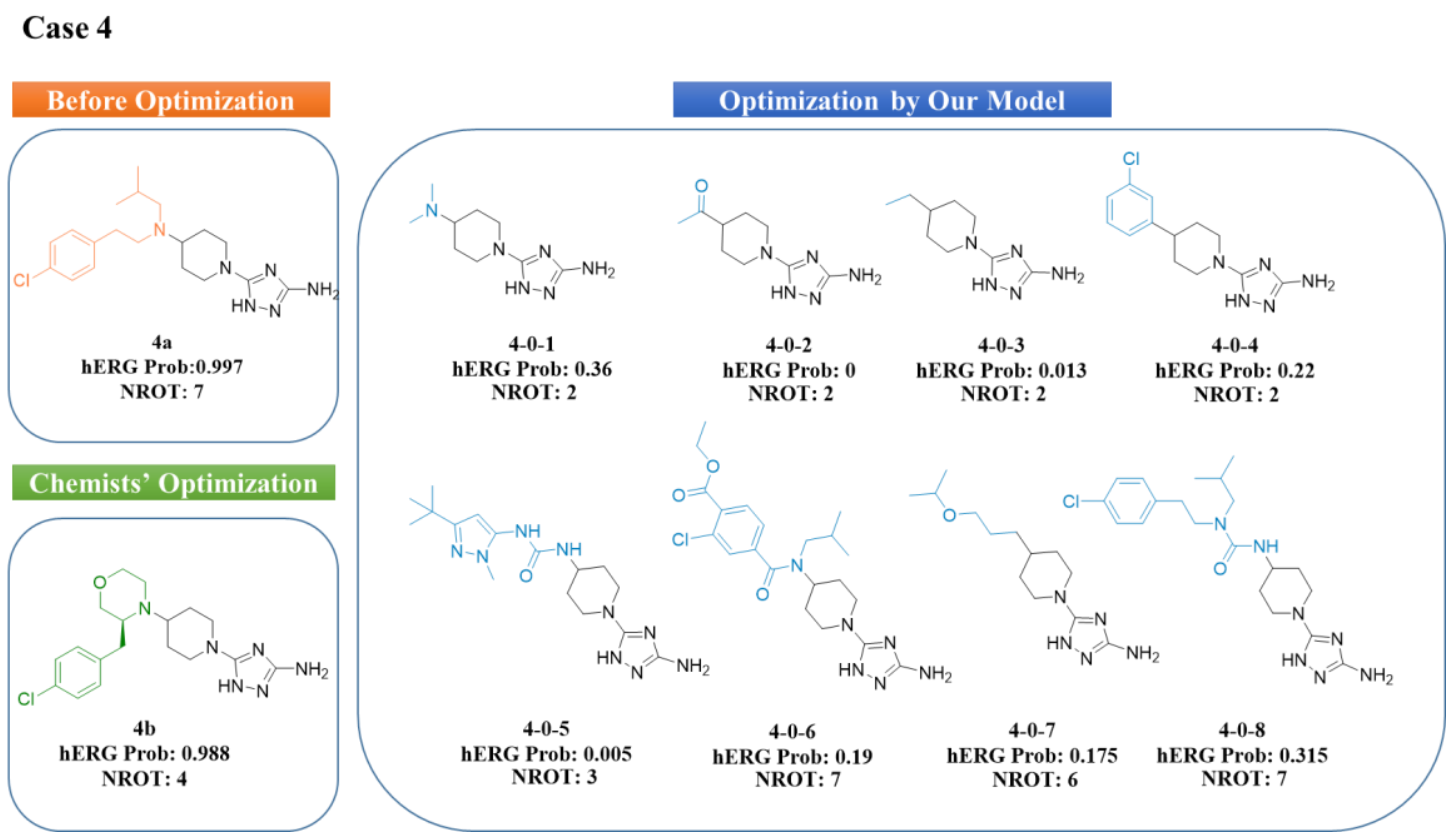

Figure 8. Examples of Optimized Structures in Case 4

Case 5. 60 epochs were carried out on core fragment 5-2 and its corresponding source fragment. Detailed information for 49 non-dupliated potential non-blockers can be seen in Table S6, and examples can be seen in Figure 9. As shown in Figure 9 and Table S6, most structures abandon the benzene ring after reinforcement learning, which agrees with the chemists' optimization strategy—altering pi-pi interaction. 5-2-1 5-26 replace the benzene with some small fragments, hERG probability of which is nearly 0 , indicating a great reduction on hERG risk. Although chemists utilized a threemembered ring together with an amide instead, their underlying regularity are similarchanging the aromatic group to the alphatic group. 5-2-7 and 5-2-8 reserve the orignal fragment except for the linker. 5-2-7 uses direct 'carbon-carbon coupling' linker while 5-2-8 adopts an amide linker. The decrease in predicted hERG-blocker probabilily 
might be caused by the position change of the aromatic ring, which may reduce the pipi interaction with the hERG channel. Case 5 shows that our model can take the action of reducing aromatic rings or chaning the postions of aromatic rings, which may help altering the pi-pi interation between compounds and hERG channel. Docking conformation comparison to the original target can be found in Figure S5, which shows that 5-2-6 can well overlap with compound $\mathbf{5 b}$.

\section{Case 5}

Before Optimization

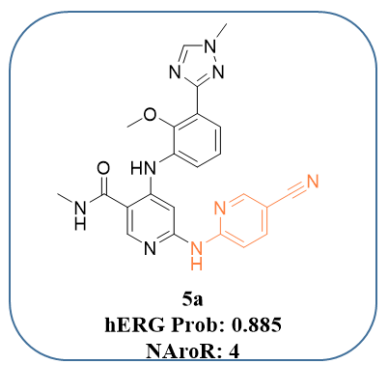

Chemists' Optimization

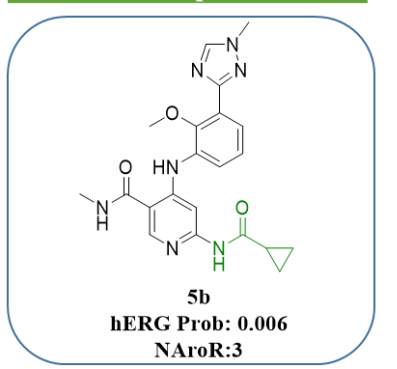

\section{Optimization by Our Model}

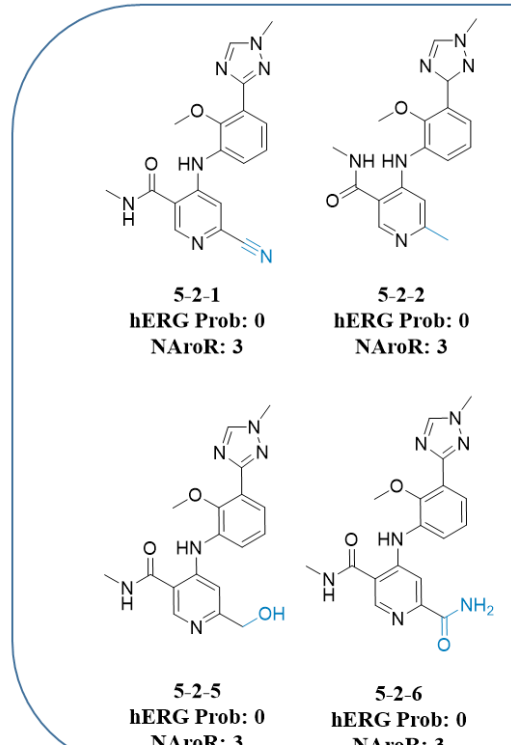

NAroR: $3 \quad$ NAroR: 3

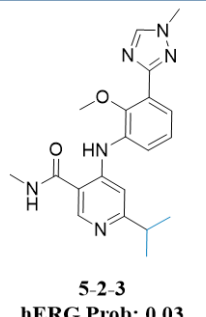

hERG Prob: 0.03

NAroR: 3

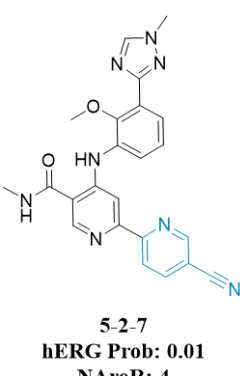

NAroR: 4

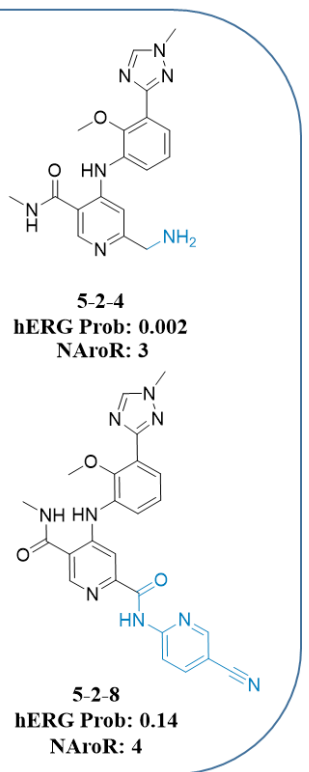

Figure 9. Examples of Optimized Structures in Case 5

\section{Conclusion}

Assessing and reducing hERG-related cardiotoxicity is a critical step in the early stage of drug discovery. In this paper, we first built eight hERG classification models based on 11,599 molecules. The selected lightGBM model shows with strong generalization ability on clinical external sets, with a BAC of 0.869 . It can still reach a BAC of 0.737 even when the similarity of compounds to the training set is less than 0.3 . Moreover, it correctly predicts five out of seven optimization cases provide by a previous study, demonstrating its ability to identify 'activity cliffs' caused by small structural changes. Then we propose a fragment growing framework termed as GGNN-RNN to reduce 
hERG binding affinity while keep particular fragments. Our model can grow more valid structures with better synthetic accessibility than naive RNN. Comparison with chemists' optimization in previous cases shows that our model can provide reliable optimizing directions including lowering lipophilicity, decreasing basicity and altering pi-pi interactions after being trained by reinforcement learning. Moreover, our model can also be used to suggest core fragments to chemists, which successfully finds out four core fragments in five cases. The hERG classification model and the GGNN-RNN model can work as a useful tool for chemists to evaluate and improve hERG property in the drug discovery pipeline.

\section{SUPPORTING INFORMATION}

The code will be uploaded to Github after our work is published.

\section{Supporting Methods}

Scheme S1 Parameters for Eight Classification Methods

Scheme S2 Equations and Explanations for Classification Metrics

Scheme S3 SMARTS of Alerts Used in This Study

\section{Supporting Tables}

Table S1 Selected Descriptors by NSGA-II

Table S2-6 Optimized Structures by Our Model in Five Cases

\section{Supporting Figures}

Figure S1 Confusion Matrix of Four Methods for External Set.

Figure S2-5 Conformation Comparison of Chemists' and Our Model's Optimization

\section{Supporting Excels}

Excel S1 Chembl Kinase Targets

\section{ABBREVIATIONS USED}


GGNN Gated Graph Neural Networks

RNN Recurrent Neural Network

NAroR NumAromaticRings

NROT NumRotatableBonds

NSGA-II Non-dominated Sorting Genetic Algorithm-II

SVM Support Vector Machine

RF Random Forest

ExtraTree Extremely Randomized Trees

Adaboost Adaptive Boosting

GBDT Gradient Boosted Decision Trees

XGBoost Exterme Gradient Boosting

LightGBM Light Gradient Boosting Machine

DNN Deep Neural Networks

AUC Area Under the ROC Curve

ACC Accuracy

BAC Balanced Accuracy

MCC Matthews Correlation Coefficient

Kappa Cohen's Kappa

F1 F1_Score

NLP Natural Language Processing

HBA The number of hydrogen acceptors

HBD The number of hydrogen donors

NROT The number of rotatable bonds

FNO The fraction of nitrogen and oxygen atoms in heavy atoms

MW Molecule weight

Alerts Pan-assay interference compounds alerts, toxic alerts and other self-

defined undesired substructures

SAR Structure-activity relationship 


\section{CORRESPONDING AUTHORS}

For Haichun Liu: Tel.: +86-25-86185163. Fax: +86-25-86185182. E-mail:

haichunliu@cpu.edu.cn

For Yadong Chen: Tel.: +86-25-86185163. Fax: +86-25-86185182. E-mail:

ydchen@cpu.edu.cn

For Lu Tao: Tel.: +86-25-86185163. Fax: +86-25-86185182. E-mail:

lutao@cpu.edu.cn

\section{Author Contributions}

The manuscript was written through contributions of all authors. All authors have given approval to the final version of the manuscript. Yan Yang and Yanmin Zhang contributed equally to this work and should be considered as co-first authors.

\section{Conflicts of interest}

The authors declare no competing financial interest.

\section{ACKNOWLEDGMENTS}

This work was financially supported by National Natural Science Foundation of China (No. 81803370), National Natural Science Foundation of China (No. 82073704), Natural Science Foundation of Jiangsu Province (No. BK20180559), State Key Laboratory Innovation Research and Cultivation Fund (No. SKLNMZZCX201812), and "Double World-classes" Construction Program of China Pharmaceutical University (No. CPU2018GF02). 


\section{Reference}

1. Craveiro, N. S.; Lopes, B. S.; Tomás, L.; Almeida, S. F., Drug Withdrawal Due to Safety: A Review of the Data Supporting Withdrawal Decision. Curr. Drug Saf. 2020, 15, 4-12.

2. Ogura, K.; Sato, T.; Yuki, H.; Honma, T., Support Vector Machine model for hERG inhibitory activities based on the integrated hERG database using descriptor selection by NSGA-II. Sci. Rep. 2019, 9, 12220 .

3. Garrido, A.; Lepailleur, A.; Mignani, S. M.; Dallemagne, P.; Rochais, C., hERG toxicity assessment: Useful guidelines for drug design. Eur. J. Med. Chem. 2020, 195, 112290.

4. Wang, S. Q.; Sun, H. Y.; Liu, H.; Li, D.; Li, Y. Y.; Hou, T. J., ADMET Evaluation in Drug Discovery. 16. Predicting hERG Blockers by Combining Multiple Pharmacophores and Machine Learning Approaches. Mol. Pharm. 2016, 13, 2855-2866.

5. Villoutreix, B. O.; Taboureau, O., Computational investigations of hERG channel blockers: New insights and current predictive models. Adv. Drug Deliv. Rev. 2015, 86, 72-82.

6. Olivecrona, M.; Blaschke, T.; Engkvist, O.; Chen, H., Molecular de-novo design through deep reinforcement learning. J. Cheminf. 2017, 9, 48.

7. Popova, M.; Isayev, O.; Tropsha, A., Deep Reinforcement Learning for de-novo Drug Design. Sci. $A d v$. 2018, 4, eaap7885

8. Jin, W.; Barzilay, R.; Jaakkola, T., Junction Tree Variational Autoencoder for Molecular Graph Generation. 2018, arXiv: 1802.04364.

9. You, J.; Liu, B.; Ying, R.; Pande, V.; Leskovec, J. In Graph Convolutional Policy Network for GoalDirected Molecular Graph Generation, NIPS, 2018.

10. Maziarka, Ł.; Pocha, A.; Kaczmarczyk, J.; Rataj, K.; Danel, T.; Warchoł, M., Mol-CycleGAN: a generative model for molecular optimization. J. Cheminf. 2020, 12 .

11. Zhou, Z.; Kearnes, S.; Li, L.; Zare, R. N.; Riley, P., Optimization of Molecules via Deep Reinforcement Learning. Sci. Rep. 2019, 9, 10752.

12. Li, Y.; Tarlow, D.; Brockschmidt, M.; Zemel, R., Gated Graph Sequence Neutral Networks. 2015, arXiv: 1511.05493

13. Li, Q.; Jørgensen, F. S.; Oprea, T.; Brunak, S.; Taboureau, O., hERG Classification Model Based on a Combination of Support Vector Machine Method and GRIND Descriptors. Mol. Pharmaceutics 2008, 5, 117-127.

14. Broccatelli, F.; Mannhold, R.; Moriconi, A.; Giuli, S.; Carosati, E., QSAR Modeling and Data Mining Link Torsades de Pointes Risk to the Interplay of Extent of Metabolism, Active Transport, and hERG Liability. Mol. Pharmaceutics 2012, 9, 2290-2301.

15. Doddareddy, M. R.; Klaasse, E. C.; Shagufta; Ijzerman, A. P.; Bender, A., Prospective validation of a comprehensive in silico hERG model and its applications to commercial compound and drug databases. ChemMedChem 2010, 5, 716-29.

16. Sun, H., An accurate and interpretable bayesian classification model for prediction of HERG liability. ChemMedChem 2006, 1, 315-22.

17. Kalyaanamoorthy, S.; Barakat, K. H., Development of Safe Drugs: The hERG Challenge. Med. Res. Rev. 2018, 38, 525-555.

18. Moriwaki, H.; Tian, Y.-S.; Kawashita, N.; Takagi, T., Mordred: a molecular descriptor calculator. $J$. Cheminf. 2018, 10, 4.

19. Schrodinger, L., Schrodinger software suite. New York: Schrödinger, LLC 2011, 670. 
20. Zhang, Y.; Chen, Y.; Zhang, D.; Wang, L.; Lu, T.; Jiao, Y., Discovery of Novel Potent VEGFR-2 Inhibitors Exerting Significant Antiproliferative Activity against Cancer Cell Lines. J. Med.Chem. 2018, 61, 140-157.

21. Daylight Chemical Information Systems Inc., 27401 Los Altos, Suite 360, Mission Viejo, CA 92691.

22. Yang, Y.; Zhang, Y.; Hua, Y.; Chen, X.; Fan, Y.; Wang, Y.; Liang, L.; Deng, C.; Lu, T.; Chen, Y.; Liu, H., In Silico Design and Analysis of a Kinase-Focused Combinatorial Library Considering Diversity and Quality. J. Chem. Inf. Model 2020, 60, 92-107.

23. Cai, C. P.; Guo, P. F.; Zhou, Y. D.; Zhou, J. W.; Wang, Q.; Zhang, F. X.; Fang, J. S.; Cheng, F. X., Deep Learning-Based Prediction of Drug-Induced Cardiotoxicity. J. Chem. Inf. Model. 2019, 59, 10731084

24. Liu, L. L.; Lu, J.; Lu, Y.; Zheng, M. Y.; Luo, X. M.; Zhu, W. L.; Jiang, H. L.; Chen, K. X., Novel Bayesian classification models for predicting compounds blocking hERG potassium channels. Acta Pharmacol. Sin. 2014, 35, 1093-102.

25. Wang, Y. W.; Huang, L.; Jiang, S. W.; Wang, Y. F.; Zou, J.; Fu, H. G.; Yang, S. Y., Capsule Networks Showed Excellent Performance in the Classification of hERG Blockers/Nonblockers. Front. Pharmacol. 2020, 10 .

26. Braga, R. C.; Alves, V. M.; Silva, M. F.; Muratov, E.; Fourches, D.; Liao, L. M.; Tropsha, A.; Andrade, C. H., Pred-hERG: A Novel web-Accessible Computational Tool for Predicting Cardiac Toxicity. Mol. Inform. 2015, 34, 698-701.

27. Didziapetris, R.; Lanevskij, K., Compilation and physicochemical classification analysis of a diverse hERG inhibition database. J. Comput. Aided Mol. Des. 2016, 30, 1175-1188.

28. Sun, H.; Huang, R.; Xia, M.; Shahane, S.; Southall, N.; Wang, Y., Prediction of hERG Liability Using SVM Classification, Bootstrapping and Jackknifing. Mol. Inform. 2017, 36.

29. Siramshetty, V. B.; Chen, Q. F.; Devarakonda, P.; Preissner, R., The Catch-22 of Predicting hERG Blockade Using Publicly Accessible Bioactivity Data. J. Chem. Inf. Model. 2018, 58, 1224-1233.

30. Lee, H. M.; Yu, M. S.; Kazmi, S. R.; Oh, S. Y.; Rhee, K. H.; Bae, M. A.; Lee, B. H.; Shin, D. S.; Oh, K. S.; Ceong, H.; Lee, D.; Na, D., Computational determination of hERG-related cardiotoxicity of drug candidates. BMC Bioinf. 2019, 20, 250.

31. Kim, H.; Nam, H., hERG-Att: Self-attention-based deep neural network for predicting hERG blockers. Comput. Biol. Chem. 2020, 87, 107286.

32. Ryu, J. Y.; Lee, M. Y.; Lee, J. H.; Lee, B. H.; Oh, K. S., DeepHIT: a deep learning framework for prediction of hERG-induced cardiotoxicity. Bioinformatics 2020, 36, 3049-3055.

33. Furber, M.; Tiden, A.-K.; Gardiner, P.; Mete, A.; Ford, R.; Millichip, 1.; Stein, L.; Mather, A.; Kinchin, E.; Luckhurst, C.; Barber, S.; Cage, P.; Sanganee, H.; Austin, R.; Chohan, K.; Beri, R.; Thong, B.; Wallace, A.; Oreffo, V.; Hutchinson, R.; Harper, S.; Debreczeni, J.; Breed, J.; Wissler, L.; Edman, K., Cathepsin C Inhibitors: Property Optimization and Identification of a Clinical Candidate. J. Med. Chem. 2014, 57, 2357-2367.

34. Wrobleski, S. T.; Moslin, R.; Lin, S.; Zhang, Y.; Spergel, S.; Kempson, J.; Tokarski, J. S.; Strnad, J.; Zupa-Fernandez, A.; Cheng, L.; Shuster, D.; Gillooly, K.; Yang, X.; Heimrich, E.; McIntyre, K. W.; Chaudhry, C.; Khan, J.; Ruzanov, M.; Tredup, J.; Mulligan, D.; Xie, D.; Sun, H.; Huang, C.; D’Arienzo, C.; Aranibar, N.; Chiney, M.; Chimalakonda, A.; Pitts, W. J.; Lombardo, L.; Carter, P. H.; Burke, J. R.; Weinstein, D. S., Highly Selective Inhibition of Tyrosine Kinase 2 (TYK2) for the Treatment of Autoimmune Diseases: Discovery of the Allosteric Inhibitor BMS-986165. J. Med. Chem. 2019, 62, 8973-8995. 
35. Du, L.; Li, M.; You, Q.; Xia, L., A novel structure-based virtual screening model for the hERG channel blockers. Biochem. Biophys. Res. Commun. 2007, 355, 889-94. 